\title{
Aislamiento por COVID-19. Un abordaje de la pandemia entre organizaciones educativas y comunitarias del Gran Buenos Aires
}

\author{
Analia Paola García ${ }^{1}$; Julieta Calderón²; Luján Rosales ${ }^{3}$; Sergio Vargas ${ }^{4}$
}

\section{Acerca de las Redes de Organizaciones Comunitarias}

La Argentina, como varios países de América Latina, ha sufrido desde los años del 70, grandes embates económicos que han impactado de manera brutal y desigual en los modos de vida de sus habitantes. Particularmente las crisis ocasionadas desde la década de los 90, con su máxima expresión en lo que se conoce como "estallido del 2001", devino en que muchos puntos del país, especialmente en el segundo y tercer cordón del conurbano bonaerense, hombres y mujeres organizaran ollas populares para dar respuesta al hambre que se produjo por los altos índices de desocupación (Svampa, 2009). Muchas de esas experiencias se han consolidado en el tiempo constituyéndose en organizaciones comunitarias que, junto a otras, han conformado Redes con fuerte anclaje territorial. En este marco nacimos los 46 centros comunitarios que conformamos la Red de Organizaciones Educativas y Comunitarias (RAE), la Red el Encuentro y la Red Andando, entre quienes articulamos proyectos y acciones que trascienden lo local, y aportamos saberes y discusiones a las políticas públicas destinadas a la niñez, y juventudes. Desarrollamos nuestras acciones en 10 distritos del Conurbano Bonaerense y en la Ciudad Autónoma de Buenos Aires. ${ }^{5}$

\section{¿Es posible pensar condiciones para un aislamiento comunitario?}

Desde el lunes 16 de Marzo, fecha en que se decretó el aislamiento social preventivo y obligatorio en el que se pedía a todos los argentinos "que se queden dentro de sus casas para evitar la propagación del COVID-19", supimos que la olla, la vianda y el vínculo con las familias, serían las formas de minimizar la circulación y los posibles contagios. Imaginábamos que gran porcentaje de las familias de los 7.800 niños $^{6}$ y jóvenes que asisten a nuestros Centros se verían imposibilitadas de sostener sus trabajos y/o de realizar las changas diarias que les permiten garantizar el ingreso familiar.

La primera semana del aislamiento, nos vimos urgidas a organizar cómo garantizar la alimentación, no sólo a quienes asisten diariamente a las actividades educativas, sino a todos los integrantes de sus familias. Posteriormente, comenzó a inquietarnos en qué condiciones transitaban los niños y jóvenes la pandemia en sus hogares, y cómo vivían la interrupción repentina de los espacios y vínculos cotidianos. Si bien recibíamos algunas noticias a través de los adultos que retiraban la comida, nos interesaba establecer un contacto con los niños y jóvenes, que nos pusiera en un diálogo e intercambio más directo y menos mediado por los adultos, para poder conocer desde sus perspectivas y vivencias, cómo estaban transitando este momento. Por lo tanto, entre educadoras y el equipo de acompañamiento

\footnotetext{
Universidad Nacional de Luján /Red Andando, centros comunitarios de educación popular. Argentina.

E-mail: analiagar1976@gmail.com

Antropóloga - Becaria Investigadora del CONICET, miembro del Equipo de Formación y acompañamiento de la RAE, Argentina.

E-mail: julietacalderonn@gmail.com

Licenciada en Políticas Públicas. Coordinadora de la Red Andando, Argentina.

E-mail: lujrosales@gmail.com

4 Docente, escritor. Miembro del Equipo de Formación de la Red el Encuentro, Argentina.

E-mail: sergiovargas2009@gmail.com

Se denomina conurbano bonaerense a las regiones que están dentro de la provincia de Buenos Aires, pero fuera de los límites de la capital federal, conformado por 8 zonas y 33 Municipios (Ley 13473/06). De acuerdo a la cercanía a CABA y su urbanización, los municipios se clasifican y ordenan en primer, segundo y tercer cordón. El censo nacional de encuestas y hogares del año 2010, arroja que el 34,9 \% de la población de la República Argentina vive en la Provincia de Buenos Aires.

6 Utilizaremos el genérico niño para referirnos tanto a niños y a niñas por razones prácticas para la lectura.
}

Soc. Infanc. 4, 2020: 185-288 
pedagógico de cada $\operatorname{Red}^{7}$ (García y Rosales, 2017; Calderón y Ferreirós, 2015) comenzamos a elaborar materiales propios, posibles de realizarse y disfrutarse, que contemplaran la diversidad de los grupos familiares y la accesibilidad tecnológica. Sabíamos que en muchos hogares había un único o ningún teléfono móvil y ninguna computadora; poca o nula conectividad a internet, imposibilidad de imprimir o fotocopiar, y principalmente mucho agobio y preocupación por la situación socioeconómica y por el exceso y dificultad para resolver las tareas escolares de hijos e hijas en la Educación Primaria o Secundaria. En ese sentido, el peso que asume para nuestra población al tener que cumplir las demandas escolares aportó un nuevo elemento de presión y frustración familiar. Los adultos tuvieron que asumir el desafío de acceder a los recursos necesarios para la "cursada a distancia", y además garantizar explicaciones, mediaciones y acompañamientos, para los cuales no estaban preparados.

A partir de una escucha atenta, y en el diálogo permanente entre educadoras y equipos de acompañamiento de nuestras Redes, definimos planificar y enviar propuestas de juego, alfabetización, matemáticas, música y cocina, para los 3.200 niños de 45 días y 5 años de edad, cuya educación inicial transcurre exclusivamente en los centros comunitarios ${ }^{8}$.

En el caso de la educación primaria, con el propósito de acompañar la resolución de tareas escolares, algunos equipos pusieron horarios fijos, por medios virtuales, para evacuar dudas. Otros implementaron distribuir de manera impresa actividades propias en los días en que se reparten los alimentos, y algunos colaboran imprimiendo las tareas que propone la escuela para garantizar mayor acceso a los materiales educativos.

\section{El aislamiento, desde la perspectiva de los niños, niñas, jóvenes y sus familias}

Puestos en marcha los modos de comunicación y envío de propuestas, comenzamos a recibir noticias de los niños y jóvenes a través de dibujos, fotografías, audios y comentarios que ellos mismos nos enviaron, y en el caso de los más pequeños mediados por los adultos o hermanos mayores.

Respecto a las edades tempranas (45 días a 5 años) notamos, a través de las fotos, una alternancia entre la participación en actividades cotidianas y en los juegos con hermanos, adultos y animales domésticos. Esas imágenes se constituyeron, para las educadoras, en la posibilidad de conocer los modos plurales en que los niños, aprenden, participan y ejercen su capacidad de agencia (Szulc, 2018). También les posibilitó afirmar que el desarrollo y los aprendizajes se pluralizan, diversifican, y complementan, en múltiples experiencias, ambientes y relaciones sociales. Además, pudieron revalorizar los aprendizajes que se llevan a cabo junto a las personas con las que transitan el aislamiento, con las cuales aprenden a partir de la colaboración y participación en actividades de la vida cotidiana. (Roggoff, 1990; Remorini, 2013). Por último, pudieron conocer y valorizar las formas particulares en que se llevan a cabo la educación y los cuidados infantiles, reconociendo su dimensión sociocultural y la complejidad que adquieren en contextos de extrema desigualdad (Colángelo, 2014; Santillán, 2012; Faur, 2014).
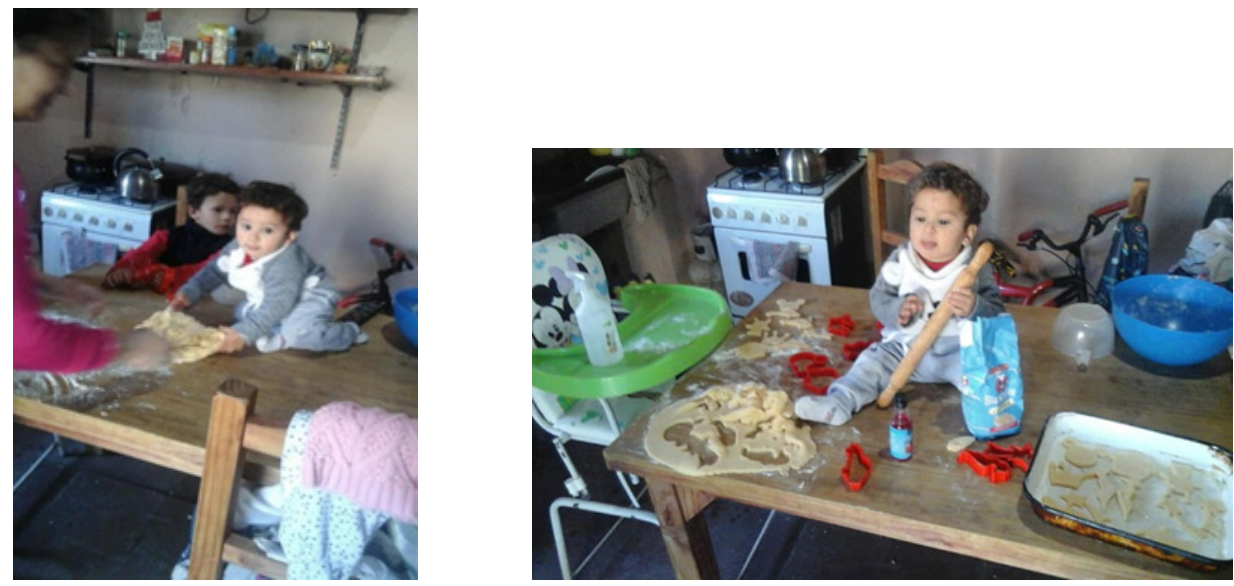

Foto 1. Familia del área maternal contándonos las actividades que realizan con su bebé. ${ }^{9}$

Las familias donde hay niños próximos a ingresar a la escuela primaria mandan fotos y videos en donde se ve la resolución de las actividades de alfabetización y matemáticas, y los modos en que los acompañan. Observamos inte-

Cada una de las Redes tiene un equipo de acompañamiento interdisciplinario propio, cuya función central es contribuir en el desarrollo de los proyectos en cada territorio.

8 De acuerdo a la Ley Nacional de Educación Nacional $N^{\circ} 26.206 / 2006$, desde los cuatros años de edad, la educación inicial es obligatoria. Sin embargo, la falta de vacantes es un problema nacional, que se acentúa en los grandes conglomerados urbanos.

Fuente: Centro Comunitario "San Cayetano de Moreno"- Red Andando. 
racciones en las cuales los adultos y niños mayores orientan, preguntan y suministran información a los más pequeños, para resolverlas. La familia de Thiago comparte algunas actividades del proyecto "Aprendo en casa". ${ }^{10}$

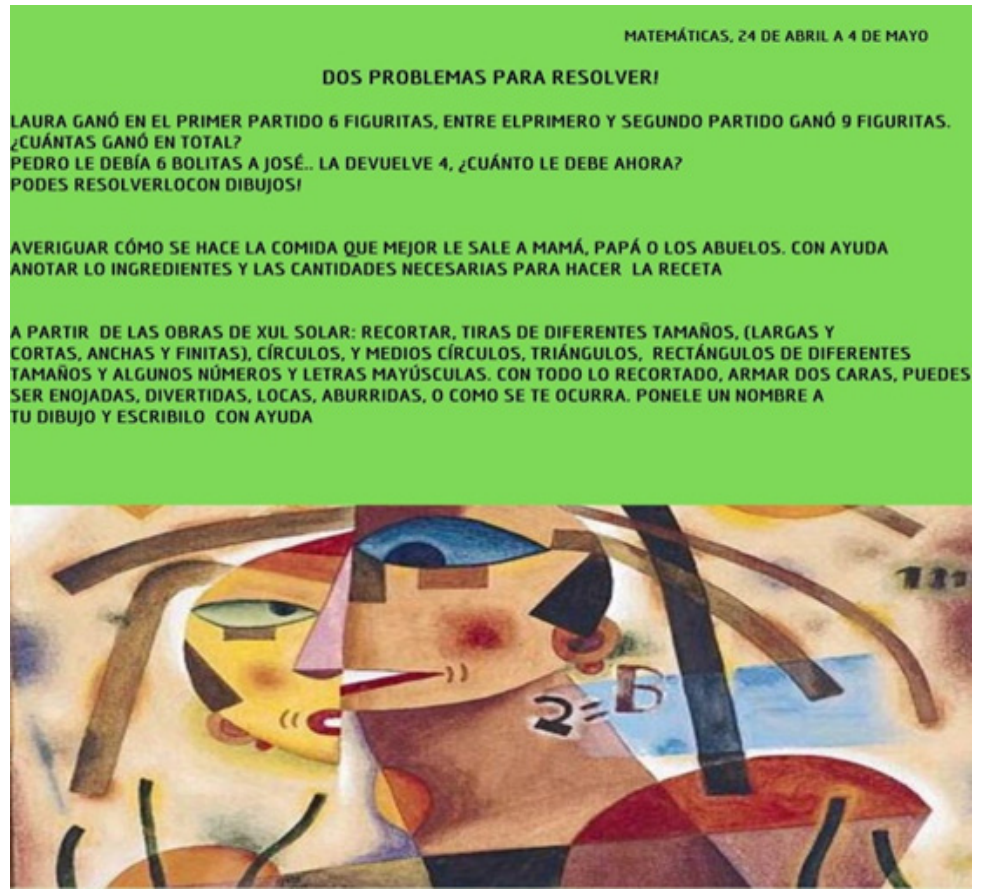

Foto 2. Flyer enviado a las familias para los niños próximos a ingresar a primer grado. ${ }^{11}$
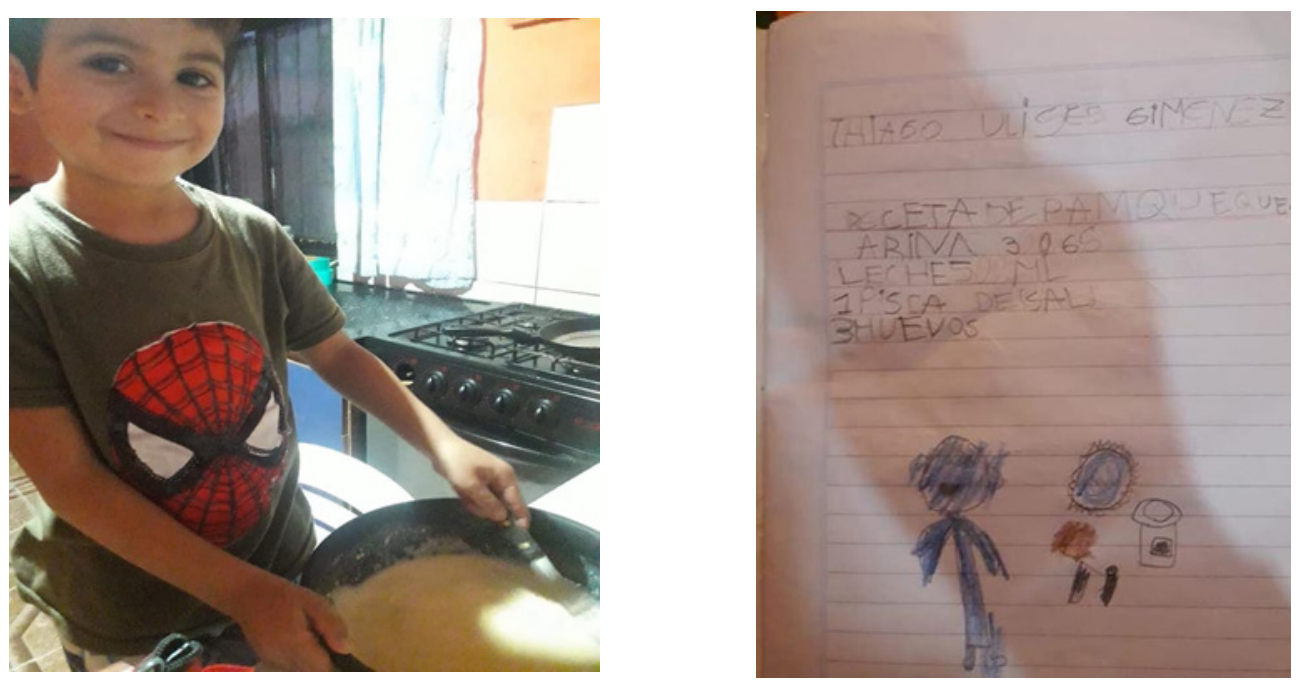

Fotos 3 y 4 . Thiago (5 años) averiguó la receta de los panqueques, los elabora y escribe los ingredientes en su cuaderno.

Respecto a los niños en edad escolar, podemos percibir la centralidad que ocupa en la organización familiar, el acompañamiento de las trayectorias escolares, en tanto la escuela es valorada y legitimada en su función de enseñanza.

A modo de ofrecer alternativas a las actividades escolares, y con la intención de propiciar espacios de participación entre los adolescentes, comenzamos a realizar programas de radio, realizados íntegramente por whatsapp, en los que se comparten saludos, canciones y reflexiones ${ }^{12}$ y preguntas que envían los niños y jóvenes. A partir de estos intercambios, los jóvenes nos expresaron:

Salgo al médico nada más. Es muy aburrido el encierro. No me mandan tanta tarea porque ando bien con las notas. Hago tareas domésticas para no aburrirme, ordeno mucho. No hay nada sucio. No extraño la escuela pero sí el

https://www.redandando.org/category/aprendo-en-casa/

11 Fuente: Red andando Proyecto "Aprendo en casa". Centro Comunitario "Casita féliz". https://www.redandando.org/category/aprendo-en-casa/

$12 \mathrm{https} / / /$ ar.ivoox.com/es/cuarenteneando-segundo-programa-audios-mp3_rf_51194711_1.html?utm_source=facebook\&utm_medium=social\&utm_ content=audio\&utm_campaign=fb_autopublish\&fbclid=IwAR2PiYlrIZgc4-C3qqQaWT3m7g458fqXtnZVj-vMrMx7qyWnWS7BGDKZK. https://youtu.be/7-VVzckqIPc/https://youtu.be/hJ5AdH8Gz4o/ 
apoyo. Espero salidas, y retomar la vida normal. El 27 tenemos la ecografía, ja ver si el bebé se deja ver! Mi fecha probable de parto es entre el 20 y el 30 de junio (Milagros, 18 años).

Me estaba por anotar a la escuela pero al final decidí dejar otra vez y ver si arranco el año que viene en la escuela para adultos. No hago mucho. De vez en cuando ordenamos en familia la casa. Hay problemas por la convivencia, pero hasta ahora pudimos arreglar cada pelea, igual. No la veo a mi novia y se hace difícil. La extraño mucho. En mi familia hay pacientes de riesgo y no salimos prácticamente. Mi papá trabaja. No me hablo mucho con amigos. Extraño salir, ir de paseo, de compras, la vida normal (Cali, 17 años).

Hemos visto adolescentes que intentan cumplir el aislamiento estricto con altos costos anímicos porque todos sus espacios de socialización y sus grupos de pertenencia, la escuela, el centro comunitario, la esquina se vieron abruptamente interrumpidos. Esto derivó en situaciones de mucha angustia expresadas incluso en ataques de pánico y gran frustración. Se suma además, para quienes están escolarizados, la presión por presentar a tiempo y bien resueltas las tareas. Marisol (17 años) es muy gráfica al expresar que siente que no está aprendiendo nada y que preferiría que suspendieran las modalidades de educación a distancia y dieran por perdido el año escolar. Un capítulo aparte nos requeriría hablar de aquellos jóvenes con consumos problemáticos, en un contexto en el que no pueden acceder a "la calle", y considerando algunos sucesos de violencia institucional por parte de las fuerzas de seguridad locales cuando se resisten a mantener una cuarentena estricta.

\section{Algunas preguntas y reflexiones en construcción}

Como decíamos al comienzo, el COVID- 19 impuso en la Argentina un aislamiento social preventivo y obligatorio, que en los barrios populares, sólo pudo cumplirse de manera comunitaria. Esta situación, en nuestro país y en el mundo entero, dejó al descubierto las desigualdades económicas, educativas y de accesibilidad a la información, en las que viven y crecen algunos niños, niñas y jóvenes de sectores populares. Además permitió problematizar y visibilizar, las tramas de relaciones e instituciones, que contribuyen a la educación y el cuidado de las infancias en el Gran Buenos Aires (Cerletti y Santillán, 2015).

La pérdida abrupta del contacto con los niños y jóvenes instaló entre educadores, la preocupación e interés en torno a cómo y dónde estarían transitando este momento. Este y otros interrogantes nos movilizaron a pensar nuevos modos de comunicación, que posibilitarían a las educadoras sortear el desconocimiento, el miedo y la resistencia que supone vincularse a través de medios tecnológicos.

A medida que fuimos restableciendo la comunicación, e intercambiamos por medio de llamadas, dibujos, fotos, videos, producidos por y con los niños, jóvenes y adultos, observamos en qué prácticas e intercambios aprenden, participan, colaboran y juegan, y el valor que le otorgan a los espacios comunitarios, "los extrañamos, queremos ir a jugar al centro, queremos jugar con nuestros amigos; necesito que las educadoras me expliquen los temas de la escuela que no entiendo". Sin embargo, los silencios de varias familias y de algunos jóvenes, generaron en los equipos incertidumbre y angustia, especialmente los de aquellos hogares con mayores dificultades económicas, con condiciones habitacionales complejas, o en donde sabemos que existe violencia de género, adultos enfermos y/o ausencia de personas que puedan garantizar cuidados y subsistencia.

Llevamos dos meses de aislamiento y por ahora no hay certezas de cuánto tiempo se prolongará y cómo será el retorno a las instituciones y actividades de la vida cotidiana. Sin embargo, considerando el actual contexto nacional y mundial, podemos prever que el regreso a las actividades presenciales no será viable en los próximos meses. En este sentido, y haciéndole frente a esta grave coyuntura que atravesamos, como educadores nos interesa avanzar en la búsqueda y creación de formas y canales que nos permitan sostener y alimentar el vínculo con los niños, jóvenes y familias, en un contexto difícil en el que educadoras y familias necesitamos seguir cerca aún en la distancia.

\section{Referencias bibliográficas}

Calderón, J., Ferreirós, F. (2015). Red de Apoyo Escolar. Hacia nuevas estrategias de intervención en el acompañamiento a las trayectorias escolares de niños, niñas y jóvenes. En Ministerio de Educación, Estudiar es tu derecho. Los espacios socioeducativos en las organizaciones comunitarias. CABA.

Colángelo, A. (2014). La crianza como proceso sociocultural. Posibles aportes de la antropología al abordaje médico de la niñez. Primeras Jornadas Diversidad en la Niñez. Hospital El Dique, Ensenada (Buenos Aires).

Cerletti, L., Santillán, L. (2015). Sobre las formas “correctas” de ser familia: lineamientos hegemónicos y prácticas cotidianas en torno a la crianza y educación infantil. 4tas Jornadas de Estudios sobre la Infancia, Buenos Aires.

Faur, E. (2014). El cuidado infantil en el siglo XXI. Mujeres malabaristas en una sociedad desigual. Buenos Aires: Siglo XXI.

García, A., Rosales, L. (2017). De Madres Cuidadoras a Educadoras Populares. Revista Voces en el Fénix, (66), 142-149 (en linea). http://www.vocesenelfenix.com/category/tema/la-educaci\%C3\%B3n-de-la-primera-infancia 
Rigal, L. (2012). Gramsci, Freire y la Educación Popular: a propósito de los nuevos Movimientos Sociales. En F. Hillert, H. Ouviña, L. Rigal, D. Suárez, Gramsci y la educación: pedagogía de la praxis y políticas culturales en América Latina. Bas. As Noveduc.

Remorini, C. (2013). Estudios etnográficos sobre el desarrollo infantil en comunidades indígenas de América Latina: contribuciones, omisiones y desafíos. Universidade Federal de Santa Catarina. Perspectiva, 31(3), 810-84.

Rogoff, B. (1990), Aprendices del Pensamiento. El desarrollo cognitivo en el contexto social. Buenos Aires: Paidós.

Santillán, L. (2012). Quiénes educan a los chicos. Infancia, trayectorias educativas y desigualdad. Buenos Aires: Biblos.

Svampa, M. (2009). Protesta, movimientos sociales y Dimensiones de la Acción Colectiva en América Latina. Ponencia en la Universidad Complutense de Madrid. España.

Szulc, A. (2019). Más allá de la agencia y las culturas infantiles. Reflexionar sobre una investigación etnográfica con niños y niñas mapuche. Revista Runa, 1(40), 53-63. 